\title{
Structure Clearance Design in Wind Tunnel Tests with Implications for Aerodynamic Drag of High- Speed Trains
}

\section{Zhixiang Huang}

China Aerodynamics Research and Development Center

Hanjie Huang ( $\square$ hansjie@hotmail.com )

China Aerodynamics Research and Development Center

\section{Weiping Zeng}

China Aerodynamics Research and Development Center

\section{Li Chen}

China Aerodynamics Research and Development Center

\section{Renyu Zhu}

China Aerodynamics Research and Development Center

\section{Research Article}

Keywords: High-speed train, Structure clearance, Aerodynamic drag, Reynolds number effect, Wind tunnel test

Posted Date: November 22nd, 2021

DOI: https://doi.org/10.21203/rs.3.rs-1073613/v1

License: (1) This work is licensed under a Creative Commons Attribution 4.0 International License. Read Full License 


\section{Abstract}

The influences of vestibule diaphragm gap, wheel-rail clearance, and strut-plate gap on the aerodynamic drag of a 1/8th-scale high-speed train model were investigated in an $8 \mathrm{~m} \times 6 \mathrm{~m}$ wind tunnel. The Reynolds number was set to $2.2 \times 10^{6}$ based on train height. It was found that the variation of the vestibule diaphragm gap changed the aerodynamic drag distribution pattern of each car; the drag coefficient of the head and middle cars might change as high as $45 \%$; however, the change in the drag coefficient of the total train was very small. The effects of the strut-plate gap on the aerodynamic drag of each car and the total train were small. The effect of the wheel-rail clearance on the drag of the head car was not significant. It was suggested that the vestibule diaphragm gap, strut-plate gap and wheel-rail clearance of the $1: 8$ scale high-speed train model should not be greater than 11,10 , and $9 \mathrm{~mm}$, respectively.

\section{Introduction}

With the rapid development of high-speed railways in East Asia and Europe, the operation speed of highspeed trains (HSTs) has reached up to $350 \mathrm{~km} / \mathrm{h}$. With increasing train speed, the aerodynamic drag of HSTs increases dramatically and accounts for nearly $80 \%$ of the total running resistance (Tian, 2009; Bell et al., 2014; Li et al., 2017). This implies that most of the energy consumed is used to overcome aerodynamic drag. A positive correlation exists between aerodynamic drag and aerodynamic noise. HSTs with lower aerodynamic drag have relatively low noise. To save energy and reduce emission, aerodynamic drag is one of the most important technical indices to evaluate the performance of HSTs with no crosswind. Therefore, finding an innovative way of reducing aerodynamic drag has become the main concern of HST manufacturers.

Streamlining the nose and tail of a train is the most effective method of achieving aerodynamic drag reduction (Baker et al., 2013; Sicot et al., 2018; Chen et al., 2019). Moreover, ensuring that the surface shape of a train is uniform and free from protuberances at inter-carriage gaps and pantograph regions is another positive approach for aerodynamic drag reduction (Mancini et al., 2001; Zhang et al., 2018). More than $24 \%$ of the total aerodynamic drag is generated from the middle cars of a train when the speed reaches $250 \mathrm{~km} / \mathrm{h}$, and the remaining aerodynamic drag comes from inter-carriage gaps, flutes at doors and windows, and outer train components (Schetz JA, 2001). It has been reported that in a 1/12th-scale train model the aerodynamic drag produced by inter-carriage structures, such as vestibule diaphragms, accounted for $4 \%$ of the total drag (Peters JL, 1980). Wind tunnel tests and numerical simulations were conducted separately to investigate the influences of different gaps on aerodynamic drag (Huang et al., 2012; Zhang et al., 2017). It was noticed that an appropriate value of vestibule diaphragm gap spacing could cause some drag reduction as compared to the closed one (gap spacing $0 \mathrm{~mm}$ ).

In the preceding aerodynamic design stage of a train, in which aerodynamic drag is a critical parameter in that stage, numerical simulation and model tests are mainly applied to investigate aerodynamic drag on HSTs. Stationary model tests have the advantages of high measurement accuracy and easy flow parameter control and are very suitable methods for the static aerodynamic characteristics evaluation 
and drag reduction assessment of prototype HSTs. In static force or pressure measurement tests, large HST models are preferred. Different from automobile tests, the moving belt technique is not used in stationary model tests due to large model lengths and requirements for ballast and track simulations. In Europe and China, fixed floors are generally used to simulate the ground effect during wind tunnel test studies on HSTs (Huang et al., 2013).

To precisely determine how much drag force is suffered by each car, the head, middle, and tail cars of a HST model should be installed independently in a wind tunnel. Forcing interference under a high wind velocity should be avoided by eliminating the contact between the cars and support system; therefore, structure clearances must be artificially introduced in train models (Fig. 1). In Fig. 1, the inner and outer vestibule diaphragms of the train model remain separate, which is referred to as the vestibule diaphragm gap, and bogie wheels maintain a certain distance from the rail, which is referred to as the wheel-rail clearance. As the support struts cross through the bottom surface of the train model, an orbicular gap always exists between each strut and the bottom plate, which is referred to as the strut-plate gap. In a 1/8th-scale HST model, the value of structure clearance varies from 3 to $20 \mathrm{~mm}$ under different test configurations, which is smaller compared to the model's characteristic dimension, but the flow field around the gap or clearance will be changed, and aerodynamics forces, especially that of most concern, namely, aerodynamic drag, will be affected.

The latest European standard (2018) has required an artificial gap between two cars of a train model. However, no specific reference values are given for the vestibule diaphragm gap, wheel-rail clearance and strut-plate gap with single-track ballast and rail configurations. Although small clearance values are always preferred, it is also necessary to avoid collisions between different parts of a train model and the support under a wind velocity of $60 \mathrm{~m} / \mathrm{s}$ above. Numerical calculations were conducted to demonstrate that the total drag coefficient of a 1/8th-scale HST model with a vestibule diaphragm gap less than 10 $\mathrm{mm}$ was equal to that of a model without a gap (Li et al., 2019). However, it was previously reported through numerical calculations that the drag coefficient discrepancy of a 1/8th-scale HST model test reached up to $6.15 \%$ (Xia et al., 2020). Very few experimental discussions on structure clearance value setting in HST train tests are available, and a limited number of experimental investigations have addressed the influences of different types of structure clearance on the aerodynamic drag of the head, middle, and tail cars of a HST train model.

Therefore, in the present work, wind tunnel tests were conducted to investigate the effects of three different types of structure clearance on the drag contribution and changing patterns of each car and the entire train. This work also aimed at finding a proper instruction on structure clearance value setting in wind tunnel tests to obtain the satisfactory aerodynamic drag of HSTs.

\section{Methodology}

\subsection{Test facility}


The current experiment was conducted in an $8 \mathrm{~m} \times 6 \mathrm{~m}$ wind tunnel at the China Aerodynamic Research and Development Center (CARDC). The test section had a length of $15 \mathrm{~m}$, width of $8 \mathrm{~m}$, height of $6 \mathrm{~m}$, and a cross-sectional area of $47.4 \mathrm{~m}^{2}$. The minimum and maximum test wind velocities were 20 and $80 \mathrm{~m} / \mathrm{s}$, respectively. The turbulence intensity of the flow was less than $0.2 \%$.

A train test platform consisting of several flat plates supported by a bracket was used in the test section (Fig. 2). A 7-m-diameter turntable was placed on the test platform and was driven synchronously with the floor turntable of the wind tunnel. The upper surface height of the test platform was set $1.06 \mathrm{~m}$ above the wind tunnel floor. When the test platform was installed, the width, height, and effective cross-sectional area of the test section were $8 \mathrm{~m}, 4.94 \mathrm{~m}$, and $39.2 \mathrm{~m}^{2}$, respectively.

Three six-component box-type force balance units (TH1004A/B/C) were used to measure the aerodynamic forces of the head, middle, and tail car models. The drag force measurement precision and accuracy of each balance were $0.03 \%$ and $0.05 \%$, respectively. A PXI system equipped with an 18-bit A/D digitizer and 128 asynchronous channels was used for data acquisition. The sampling frequency was $200 \mathrm{~Hz}$ and the data sampling time $30 \mathrm{~s}$.

\subsection{Test model}

Generally, 1/8th-scale HST models are used for aerodynamic research works of China railway high-speed (CRH) trains. In the present study, CRH-A, CRH-B, and CRH-C models at a scale ratio of 1:8 were used (Fig. 3). Each train model was designed with bogies at the bottom and without a pantograph on the top. The outside body of the models was shaped by synthetic resin plates, and their inside structure was built with a metal frame. The accuracy of the model shape was set to the maximum tolerance of $0.05 \%$. Joint plates for force balance mounting were welded on the inside frame of the car models. A force balance unit was jointed with the model body by a joint plate on the upper surface of the unit, and it was jointed with ballasts by a four-strut support on its lower surface.

Two different train car formations were adopted in the current research (Fig. 4). The first consisted of the head, middle, and tail cars of CRH-A or CRH-B. The total length $(L)$ of this three-car formation was 9.972 $\mathrm{m}$ without considering the inter-carriage gap between two cars, and its width $(B)$, height $(H$, defined as the characteristic length), and cross-sectional area were $0.418 \mathrm{~m}, 0.472 \mathrm{~m}$, and $0.1886 \mathrm{~m}^{2}$, respectively. The second formation consisted of the head car and the half of the middle car of $\mathrm{CRH}-\mathrm{C}$, and its length, width, height, and cross-sectional area were $4.986 \mathrm{~m}, 0.418 \mathrm{~m}, 0.472 \mathrm{~m}$, and $0.1886 \mathrm{~m}^{2}$, respectively. Ballasts with lengths of 14.3 and $7.7 \mathrm{~m}$ were installed on the platforms of the three-car and one-and-a-half-car formations, respectively, and a track model was located on the ballast surface. Specific transition slopes and arcs were adopted for the two ends of the ballast. The slope of the front and back ends was $15^{\circ}$, and the slope of both sides was $35^{\circ}$.

\subsection{Experimental scheme}

In the present work, aerodynamic force measurement tests were conducted with a yaw angle $(\beta)$ of $0^{\circ}$ and a wind velocity of $70 \mathrm{~m} / \mathrm{s}$. 
Non-zero yaw angles have great influences on aerodynamic force; however, it should be noted that in most cases, HSTs act in a straight-line running state with much smaller crosswind speed compared with the train running speed. This kind of running state is also the fundamental state in HST research; thus, in wind tunnel tests, the yaw angle of a train model is generally set to $0^{\circ}$.

The similarity criterion for HST wind tunnel tests is mainly Reynolds number similarity. According to Bell et al. (2014) and The CEN European Standard (2009), HST wind tunnel tests should be conducted with separate Reynolds numbers greater than $1.0 \times 10^{6}$ or $2.5 \times 10^{5}$. It is believed that flow structures do not change significantly when the Reynolds number is greater than $1.0 \times 10^{6}$. The average wind velocity for the current experiment was $70 \mathrm{~m} / \mathrm{s}$, and the corresponding Reynolds number based on the train model height was $2.2 \times 10^{6}$; thus, the Reynolds number similarity for wind tunnel tests was satisfied.

To study the influences of vestibule diaphragm gap (VDG) and strut-plate gap (SPG), the three-car formation models of $\mathrm{CRH}-\mathrm{A}$ and $\mathrm{CRH}-\mathrm{B}$ were used. Five different vestibule diaphragm gaps were used-4, $7,11,16$, and $21 \mathrm{~mm}$-and the corresponding schemes were termed VDG-1, VDG-2, VDG-3, VDG-4, and VDG-5, respectively. The inner and outer vestibule diaphragm gaps maintained the same size. Four different strut-plate gaps were used $-3,6,10$, and $15 \mathrm{~mm}$-and the corresponding schemes were termed SPG-1, SPG-2, SPG-3, and SPG-4, respectively.

To study the influences of wheel-rail clearance (WRC), the one and a half-car formation model of CRH-C was used. Five different wheel-rail clearances were used-3, 5, 7, 9, and $12 \mathrm{~mm}$-and the corresponding schemes were termed WRC-1, WRC-2, WRC-3, WRC-4, and WRC-5, respectively. It should be noted the actual height of the car model was raised simultaneously with the increase of WRC.

Nearly 100 combinations of VDG, SPG, and WRC are available. To make the combinatory analysis realistic, when one parameter was changed, the other two parameters were kept unchanged. When the vestibule diaphragm gap varied from 4 to $22 \mathrm{~mm}$, the same size $(5 \mathrm{~mm})$ was used for the strut-plate gap and wheel-rail clearance. When the strut-plate gap varied from 3 to $15 \mathrm{~mm}$, the same size was $(5 \mathrm{~mm})$ used for the vestibule diaphragm gap and wheel-rail clearance, while when the wheel-rail clearance varied from 3 to $12 \mathrm{~mm}$, the same size $(5 \mathrm{~mm})$ was used for the vestibule diaphragm gap and strut-plate gap.

Aerodynamic forces, mainly drag forces on head, middle, and tail cars, were normalized nondimensionally by Eq. (1) according to the European Standard (2009 and 2010).

$$
C_{X}^{\prime}=\frac{F_{X}}{\frac{1}{2} \rho V_{r e f}^{2} S}
$$

1

where $F_{X}$ is the drag force of the head, middle, or tail cars; $C_{X}^{\prime}$ is the drag coefficient of the head, middle, or tail cars without data correction; $S$ is the reference area of the model $\left(0.1856 \mathrm{~m}^{2}\right) ; V_{\text {ref }}$ is the average speed of incoming airflow; and $\rho$ is air density $\left(1.2 \mathrm{~kg} / \mathrm{m}^{3}\right)$. 
The maximum blockage ratio was less than $1 \%$ at $\beta=0^{\circ}$; thus, no blockage correction needed to be conducted. The horizontal buoyancy effect of each car was modified by Eq. (2) according to the axial static pressure gradient in the area where each car was located:

$$
C_{X}=C_{X}^{\prime}+L \cdot d C_{p} / d x
$$

2

where $C_{X}$ is the modified coefficient of aerodynamic drag, $L$ the car length, and $d E_{p} / d x=-0.0052$ is the axial static pressure gradient and was obtained by experimental calibration.

\section{Results And Analysis}

\subsection{Effects of Reynolds number}

Reynolds number greatly affected the boundary layer thickness on the HST surface, resulting in a change of the aerodynamic force. According to Bell et al. (2014), the HST model tests were conducted at critical Reynolds number greater than $1.0 \times 10^{6}$ to ensure that the drag coefficient changed insignificantly with the increase of the Reynolds number. For wind velocities of $30,35,40,45,50,55,60,65$, and $70 \mathrm{~m} / \mathrm{s}$, the corresponding Reynolds numbers were $9.44 \times 10^{5}, 1.10 \times 10^{6}, 1.26 \times 10^{6}, 1.42 \times 10^{6}, 1.57 \times 10^{6}, 1.73 \times$ $10^{6}, 1.89 \times 10^{6}, 2.05 \times 10^{6}$ and $2.20 \times 10^{6}$, respectively. Reynolds number was calculated by Eq. (3):

$$
\operatorname{Re}=\frac{\rho V_{r e f} H}{\mu}
$$

3

where $\mathrm{H}$ is the height of the head car model $(0.472 \mathrm{~m})$ and $\mu$ is the air viscosity coefficient $\left(1.8 \times 10^{-5}\right.$ Pa.s).

It is evident from Fig. 5 and Table 1 that the changes in the drag coefficients of the HST head car models of $\mathrm{CRH}-\mathrm{A}, \mathrm{CRH}-\mathrm{B}$, and $\mathrm{CRH}-\mathrm{C}$ were small at different wind velocities from 30 to $70 \mathrm{~m} / \mathrm{s}$. The drag coefficient error ranges for $\mathrm{CRH}-\mathrm{A}, \mathrm{CRH}-\mathrm{B}$, and $\mathrm{CRH}-\mathrm{C}$ HST models were $3.52 \%, 1.85 \%$, and $2.12 \%$, respectively, and the drag coefficients of these three head cars changed very little in the wind speed range $50-70 \mathrm{~m} / \mathrm{s}$. The impacts of wind velocity on the drag coefficients of the head, middle, and tail cars were insignificant when the wind velocity was larger than $55 \mathrm{~m} / \mathrm{s}$. Hence, in the following experiment, the wind velocity of $70 \mathrm{~m} / \mathrm{s}$ was selected as the test velocity. 
Table 1

Drag coefficients of head car of CRH-A, CRH-B, and CRH-C

\begin{tabular}{|c|c|c|c|c|c|c|c|c|c|c|}
\hline & $\begin{array}{l}30 \\
\mathrm{~m} / \mathrm{s}\end{array}$ & $\begin{array}{l}35 \\
\mathrm{~m} / \mathrm{s}\end{array}$ & $\begin{array}{l}40 \\
\mathrm{~m} / \mathrm{s}\end{array}$ & $\begin{array}{l}45 \\
\mathrm{~m} / \mathrm{s}\end{array}$ & $\begin{array}{l}50 \\
\mathrm{~m} / \mathrm{s}\end{array}$ & $\begin{array}{l}55 \\
\mathrm{~m} / \mathrm{s}\end{array}$ & $\begin{array}{l}60 \\
\mathrm{~m} / \mathrm{s}\end{array}$ & $\begin{array}{l}65 \\
\mathrm{~m} / \mathrm{s}\end{array}$ & $\begin{array}{l}70 \\
\mathrm{~m} / \mathrm{s}\end{array}$ & $\begin{array}{l}\text { Error } \\
\text { range }\end{array}$ \\
\hline $\begin{array}{l}\text { CRH- } \\
\text { A }\end{array}$ & 0.070 & 0.067 & 0.067 & 0.066 & 0.066 & 0.064 & 0.064 & 0.063 & 0.062 & $3.52 \%$ \\
\hline $\begin{array}{l}\mathrm{CRH}- \\
\mathrm{B}\end{array}$ & 0.129 & 0.126 & 0.126 & 0.125 & 0.124 & 0.123 & 0.123 & 0.122 & 0.121 & $1.85 \%$ \\
\hline $\begin{array}{l}\mathrm{CRH}- \\
\mathrm{C}\end{array}$ & 0.113 & 0.110 & 0.110 & 0.109 & 0.108 & 0.107 & 0.107 & 0.106 & 0.105 & $2.12 \%$ \\
\hline
\end{tabular}

\subsection{Repeatability precision of force measurement}

Five repeated force measurement tests of the three-car formation were conducted at $70 \mathrm{~m} / \mathrm{s}$ and $\beta=0^{\circ}$ when the vestibule diaphragm gap and strut-plate gap schemes were VDG-1 and SPG-2, respectively. The root-mean-square (RMS) values of the drag coefficients of the head, middle, and tail cars and the total train were $0.0004,0.0003,0.0006$, and 0.0005 , respectively, and the corresponding limited errors were $0.0012,0.0009,0.0018$, and 0.0015 , respectively. For the strut-plate gap scheme of SPG-2, the RMS values of the drag coefficients of the head, middle, and tail cars and the total train were $0.0005,0.0004$, 0.0005 , and 0.0006 , respectively, and the corresponding $95 \%$ confidence uncertainty limited errors were $0.0015,0.0012,0.0015$, and 0.0018 , respectively. In addition, five repeated force measurement tests were conducted for the one-and-a-half-car formation under the wheel-rail clearance scheme of WRC-2. The RMS value of the drag coefficient of the head car was 0.0007 , and the corresponding limited error was 0.0021 . The installation angle errors of yaw, overthrow, and pitching of the train model were all less than $0.05^{\circ}$. Therefore, it can be inferred that the repeatability precision of the HST force measurement tests was comparable with airplane wind tunnel tests.

\subsection{Influence of vestibule diaphragm gap on aerodynamic drag}

The aerodynamic drag coefficients of each car and the total train of CRH-A for different vestibule diaphragm gap are presented in Table 2 and Fig. 6.

The influence of VDG spacing on the change of $C_{X-h e a d}$ was much smaller than that of $C_{X \text {-middle }}$ and $C_{x-\text { tail }}$

The drag coefficients for the VDG of $4 \mathrm{~mm}$ were selected as the standard values for comparison. When the VDG increased from 4 to $21 \mathrm{~mm}$, the drag coefficient of the total train $\left(C_{x \text {-total }}\right)$ increased by approximately $2.29 \%$, and when VDG was $11 \mathrm{~mm}$, the increment of $C_{x \text {-total }}$ was only $0.5 \%$. Therefore, it can be inferred that the influence of VDG on the total aerodynamic drag of the three-car train was relatively small. However, the existence of VDG changed the drag force distribution pattern among the three cars of the train. The drag coefficients of the head car $\left(C_{x \text {-head }}\right)$ and tail car $\left(C_{x \text {-tail }}\right)$ increased and 
decreased, respectively, with the increase of the VDG. The drag coefficient of the middle car $\left(C_{x-m i d d l e}\right)$ increased with the increasing VDG to $16 \mathrm{~mm}$ and then decreased dramatically when the spacing increased to $21 \mathrm{~mm}$. When the spacing value was $21 \mathrm{~mm}$, the head car suffered a sudden drag increase of approximately $45 \%$. This bullish-bearish change phenomenon might happen because the airflow easily passed through the inter-car gap and changed the flow field between the head and middle cars when VDG was large. This bullish-bearish change of $C_{x \text {-head }}$ and $C_{x \text {-middle }}$ is different from previous numerical simulation results of Li et al. (2019) and Xia et al. (2020), in which there were not sudden changes for the $C_{x \text {-head }}$ and $C_{x \text {-middle. }}$. Except for the spacing of $22 \mathrm{~mm}$, the influence of VDG on $C_{x \text {-head }}$ was much smaller than those of $C_{x \text {-middle }}$ and $C_{x \text {-tail. }}$

Table 2

Drag coefficients of each car and total train of $\mathrm{CRH}-\mathrm{A}$ for different vestibule diaphragm gaps

\begin{tabular}{|llllll|}
\hline Scheme & spacing & \multicolumn{2}{l}{$C_{\boldsymbol{x}}$} & & \\
& & Head car & Middle car & Tail car & Total train \\
\hline VDG-1 & $4 \mathrm{~mm}$ & 0.062 & 0.105 & 0.226 & 0.393 \\
\hline VDG-2 & $7 \mathrm{~mm}$ & 0.064 & 0.113 & 0.217 & 0.394 \\
\hline VDG-3 & $11 \mathrm{~mm}$ & 0.066 & 0.128 & 0.201 & 0.395 \\
\hline VDG-4 & $16 \mathrm{~mm}$ & 0.070 & 0.140 & 0.187 & 0.397 \\
\hline VDG-5 & $21 \mathrm{~mm}$ & 0.102 & 0.118 & 0.182 & 0.402 \\
\hline
\end{tabular}

When VDG was in the range 4-21 mm and the dimensionless distance parameter (VDG/B) was in the range $0.01-0.05$, the drag coefficients of the head car, middle, and tail cars and the total car were fitted using cubic or quadratic polynomials (Fig. 7).

The influences of VDG on aerodynamic drag force distribution patterns were remarkable; however, the variation of $C_{x-\text { total }}$ was small. This happened because the change of VDG led to a change of the flow field around the vestibule diaphragm and in the static pressure in the cavity of the vestibule diaphragm. The drag distribution patterns of each car and the entire train changed due to the variation of pressure drag. However, the pressure drag acted as an "internal force" and had little influence on the aerodynamic drag of the total train. Therefore, it was recommended that the vestibule diaphragm gap of the 1:8 scale HST model should be less than $11 \mathrm{~mm}$, and this inference is in accordance with the numerical simulation result of Li et al. (2019). However, an phenomenon opposite that in present work was observed (Zhang et al., 2017); that is-with the increase of the outer VDG, the aerodynamic drag of the head car and tail cars decreased and increased, respectively. This might have happened because the inner-vestibule diaphragm gap was not considered in the literature (Zhang et al., 2017), and the specific structure of the vestibule diaphragm was different from that of the present work.

\subsection{Influences of strut-plate gap on aerodynamic drag}


The aerodynamic drag coefficients of each car and the total train for different strut-plate gaps are presented in Table 3 and Fig. 8.

Table 3

Drag coefficients of each car and total train for different strut-plate gaps

\begin{tabular}{|llllll|}
\hline Scheme & Spacing & \multicolumn{2}{l}{$C_{\boldsymbol{x}}$} & & \\
\cline { 3 - 6 } & & Head car & Middle car & Tail car & Total train \\
\hline SPG-1 & $3 \mathrm{~mm}$ & 0.121 & 0.086 & 0.180 & 0.387 \\
\hline SPG-2 & $6 \mathrm{~mm}$ & 0.122 & 0.087 & 0.181 & 0.390 \\
\hline SPG-3 & $10 \mathrm{~mm}$ & 0.121 & 0.088 & 0.181 & 0.390 \\
\hline SPG-4 & $15 \mathrm{~mm}$ & 0.120 & 0.087 & 0.179 & 0.386 \\
\hline
\end{tabular}

When the SPG increased from 3 to $15 \mathrm{~mm}$, the drag coefficients of the head car $\left(C_{x \text {-head }}\right)$, middle $\left(C_{x-\text { middle }}\right)$, and tail cars $\left(C_{x \text {-tail }}\right)$ and the total train $\left(C_{x \text {-total }}\right)$ changed very little (less than $\left.1 \%\right)$. This happened because the SPG was in the boundary layer of the bottom of the train body, where the airflow velocity was very small. Therefore, it can be inferred that the change of SPG had little influence on the flow pattern. Hence, it was recommended that the strut-plate gap of the 1:8 scale HST model should not be greater than $10 \mathrm{~mm}$.

\subsection{Influences of wheel-rail clearance on aerodynamic drag}

The aerodynamic drag coefficients of the head car for different wheel-rail clearance values are presented in Table 4 and Fig. 9.

Table 4

Drag coefficients of head car for different wheel-rail clearance values

\begin{tabular}{|lll|}
\hline Scheme & Clearance size & $C_{\boldsymbol{x}}$ \\
\cline { 3 - 3 } & & Head car \\
\hline WRC-1 & $3 \mathrm{~mm}$ & 0.105 \\
\hline WRC-2 & $5 \mathrm{~mm}$ & 0.106 \\
\hline WRC-3 & $7 \mathrm{~mm}$ & 0.108 \\
\hline WRC-4 & $9 \mathrm{~mm}$ & 0.109 \\
\hline WRC-5 & $12 \mathrm{~mm}$ & 0.112 \\
\hline
\end{tabular}

When the WRC increased from 3 to $12 \mathrm{~mm}$, the increment of the drag coefficient of the head car $\left(C_{x-h e a d}\right)$ was approximately $7 \%$. It should be pointed out that when the WRC increased to $7 \mathrm{~mm}$, the increment of $C_{x \text {-head }}$ was below $3 \%$. Therefore, it can be inferred that the influence of the WRC on the aerodynamic drag of the train head car was not significant. 
With the increase of the wheel-rail clearance, the height of the train body from the ballast surface also increased. In the present work, the ballast was used to simulate the fixed ground, and a boundary layer existed on the ballast surface.

The boundary layer thickness gradually increased from the front edge to the tail end of the ballast, and the head car, especially its tail end, was partially "submerged" into the boundary layer. Considering the wind speed profile of the boundary layer, when the train body height was slightly raised, the flow passage tunnel between the bottom of the train body and the upper surface of the ballast slightly expanded, thus accelerating the airflow. The "submerged" part of the head car encountered the incoming airflow with a relatively larger velocity; thereby, the aerodynamic drag of the head car increased slightly. Hence, it was recommended that the wheel-rail clearance of the 1:8 scale model should not be greater than $7 \mathrm{~mm}$.

\section{Conclusions}

In the present research, wind tunnel tests of a 1:8 scale HST model were carried out, and the effects of vestibule diaphragm gap, strut-plate gap and wheel-rail clearance on the aerodynamic drag of the HST model at zero yaw angle were investigated. The main observations are the following:

1. The variation of the vestibule diaphragm gap changed the aerodynamic drag distribution pattern of each car; however, it had little influence on the drag of the total train. The vestibule diaphragm gap of the $1: 8$ scale HST model should be less than $11 \mathrm{~mm}$.

2. The variation of the strut-plate gap had little influence on the aerodynamic drag of each car and the total train. It was suggested that the strut-plate gap of the 1:8 scale HST model should not be greater than $10 \mathrm{~mm}$.

3. The influence of wheel-rail clearance on the aerodynamic drag of the train head car was not significant. The wheel-rail clearance of the 1:8 scale HST model should be less than $9 \mathrm{~mm}$.

The obtained results can provide a useful reference for HST model simulations with necessary manmade gap and clearance in wind tunnel tests and can also be used as supplementary data to the EN14067-6 (2018) standard. If the ground state of this experiment is different, such as a moving belt being used to simulate the moving ground state, the influences of wheel-rail clearance and strut-plate gap on the test results must be further studied.

It should be noticed that only individual analysis for the three influencing factors were conducted in current study. In author's opinion, when three kinds of clearances must be simulated at the same time, priority should be given to the vestibule diaphragm gap. As indicated in Tables $2-4$, for the aerodynamic design of the shape of head car, the vestibule diaphragm gap is the factor of most concern $s$ in tests. The coupling mechanism between these multiple factors still needs further investigation, and combinatorial analysis based on large amounts of test conditions and data should be the next stage work.

\section{Declarations}




\section{Acknowledgements}

This work was supported by the National Natural Science Foundation of China (Grant No. 52078437). We thank LetPub (www.letpub.com) for linguistic assistance.

\section{References}

1. Baker, C. J., Quinn, A., Sima, M., Hoefener, L. \& Licciardello, R. (2013) Full-scale measurement and analysis of train slipstreams and wakes. Part 2: Gust Analysis. Proc. Inst. Mech. Eng. - Part F J. Rail Rapid Transit 228:468-480, DOI: 10.1177/0954409713485944

2. Bell, J. R., Burton, D., Thompson, M. C., Herbst, A. H. \& Sheridan, J. Wind tunnel analysis of the slipstream and wake of a HST. Journal of Wind Engineering \& Industrial Aerodynamics, 134, 122138 (2014).

3. CEN European Standard. EN 14067-6:2009, Railway applications-aerodynamics Part 4: requirements and test procedures for aerodynamics on open track (CEN CEN-CENELEC Management Centre, 2009).

4. CEN European Standard. EN 14067-6:2010, Railway applications-aerodynamics Part 6: requirements and test procedures for cross wind assessment (CEN-CENELEC Management Centre, 2010).

5. CEN European Standard. EN 14067-6:2018, Railway applications-aerodynamics. Part 6: requirements and test procedures for cross wind assessment (CEN-CENELEC Management Centre, 2018).

6. Chen, G. et al. Dynamic analysis of the effect of nose length on train aerodynamic performance. Journal of Wind Engineering \& Industrial Aerodynamics, 184, 198-208 (2019).

7. Huang, Z. X., Chen, L. \& Jiang, K. L. Influence of length of train formation and vestibule diaphragm structure on aerodynamic drag of high speed train model. Journal of Experiments in Fluid Mechanics, 26 (5), 36-41 (2012). (in Chinese)

8. Huang, Z. X., Chen, L. \& Zhang, W. Z. Study on simulation manner of wind tunnel test of HST model. Journal of Railway Science and Engineering, 10 (3), 87-92 (2013). (in Chinese)

9. Li, T., Li, M., Wang, Z. \& Zhang, J. Y. (2019) Effect of the inter-car gap length on the aerodynamic characteristics of a high-speed train. Proceedings of the Institution of Mechanical Engineers, Part F: Journal of Rail and Rapid Transit 233(4):448-465, DOI: 10.1177/0954409718799809

10. Li, Z. W., Yang, M. Z., Huang, S. \& Liang, X. F. A new method to measure the aerodynamic drag of HSTs passing through tunnels. Journal of Wind Engineering \& Industrial Aerodynamics, 171, 110120 (2017).

11. Mancini, G. et al. (2001) Effects of experimental bogie fairings on the aerodynamic drag of the ETR 500 high speed train. In: Proceedings of the World Congress on Railway Research, 25-29 November, Koln, Germany

12. Peters, J. L. (1980) Optimising aerodynamics to raise IC performance.Railway Gazette International10:817-819 
13. Schetz, J. A. Aerodynamics of High-Speed Trains. Annual Review of Fluid mechanics, 33, 371-414 (2001).

14. Sicot, C., Deliancourt, F., Boree, J., Aguinagab, S. \& Bouchetb, J. P. Representativeness of geometrical details during wind tunnel tests, Application to train aerodynamics in crosswind conditions. Journal of Wind Engineering \& Industrial Aerodynamics, 177, 186-196 (2018).

15. Tian, H. Q. Formation mechanism of aerodynamic drag of HST and some reduction measures. J. Cent. S. Univ, 16 (1), 166-171 (2009).

16. Xia, Y. T. et al. Aerodynamic effects of the gap spacing between adjacent vehicles on wind tunnel train models. Engineering Applications of Computational Fluid Mechanics, 14 (1), 835-852 (2020).

17. Zhang, J., Wang, J. B., Wang, Q. X., Xiong, X. H. \& Gao, G. J. A study of the influence of bogie cut outs' angles on the aerodynamic performance of a HST. Journal of Wind Engineering \& Industrial Aerodynamics, 175, 153-168 (2018).

18. Zhang, Y., Sun, Z. X., GUO, D. L., YANG, G. W. \& SHANG KM Effects of Windshield Slot Width on Aerodynamics of High-speed Trains. Journal of the China Railway Society, 39 (3), 19-24 (2017). (in Chinese)

\section{Figures}




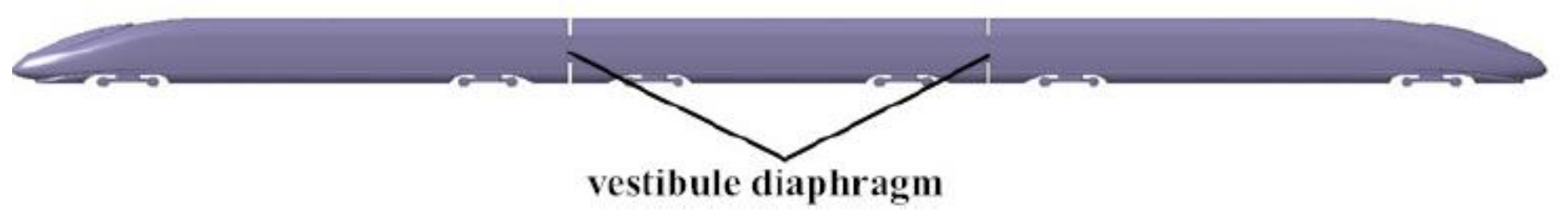

(a) Vestibule diaphragm gap

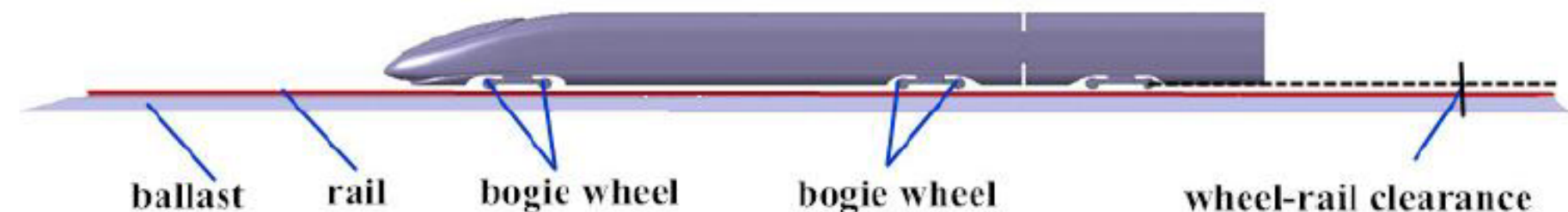

(b) Wheel-rail clearance

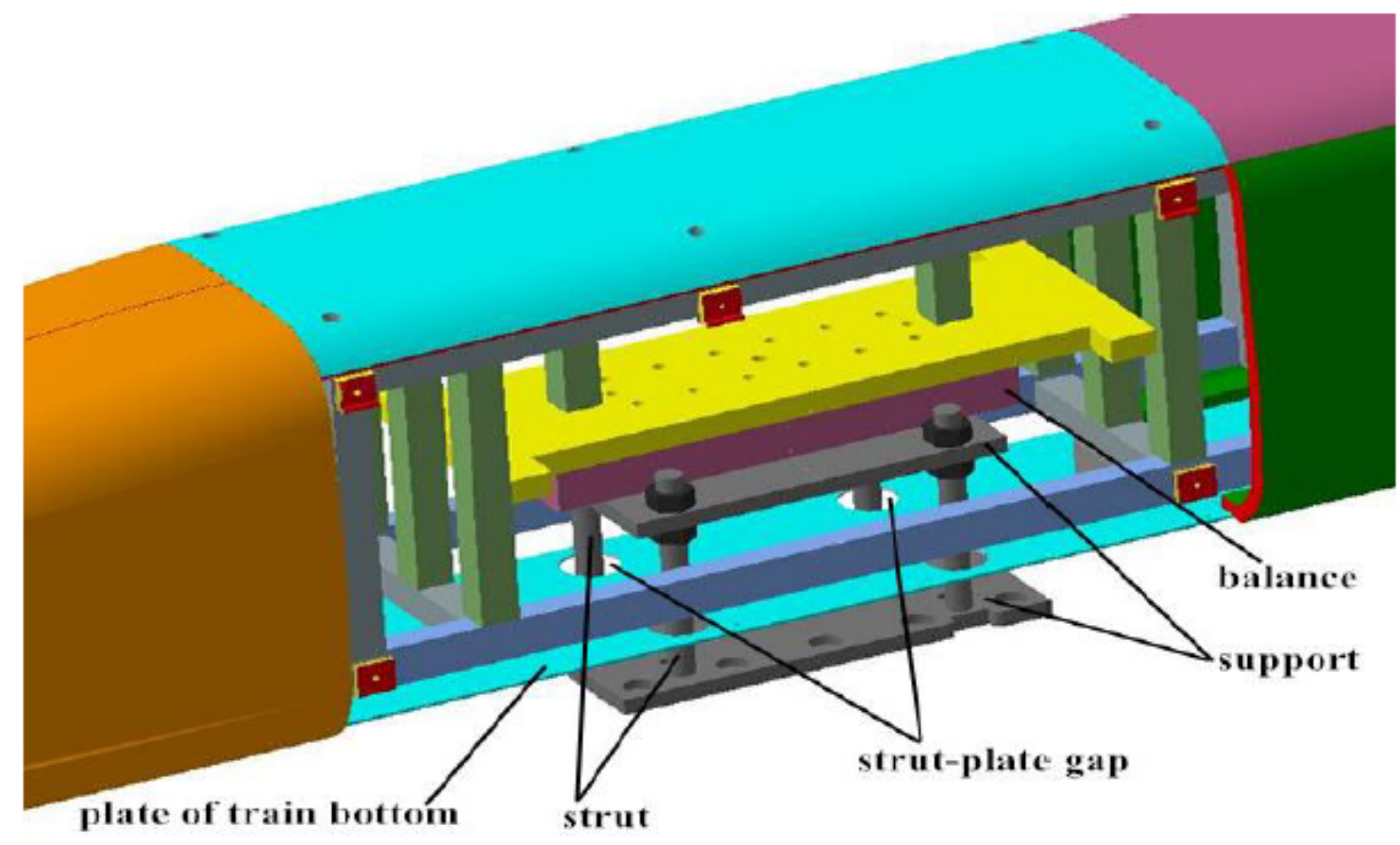

(c) Strut-plate gap

Figure 1

Structure clearance of HST model in wind tunnel test 


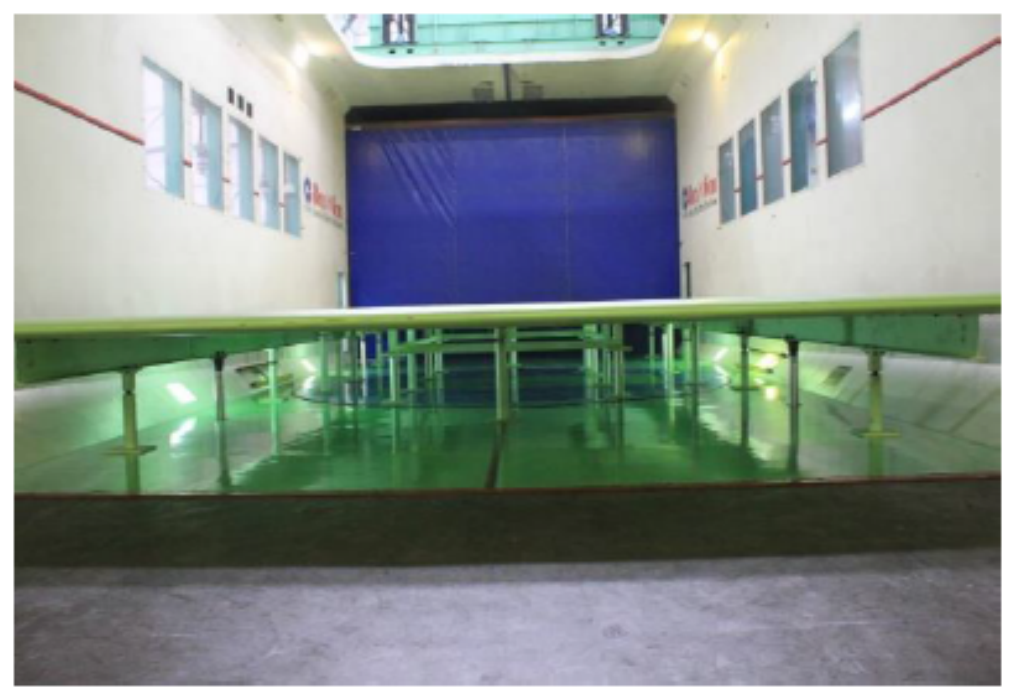

(a)

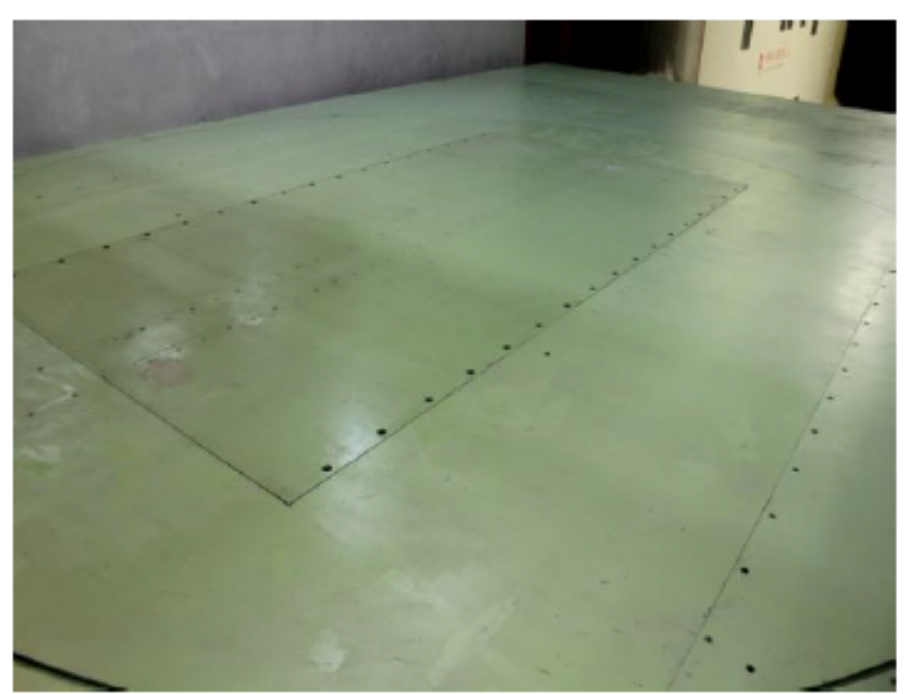

(b)

Figure 2

Train test platform: (a) bracket and (b) flat plates 


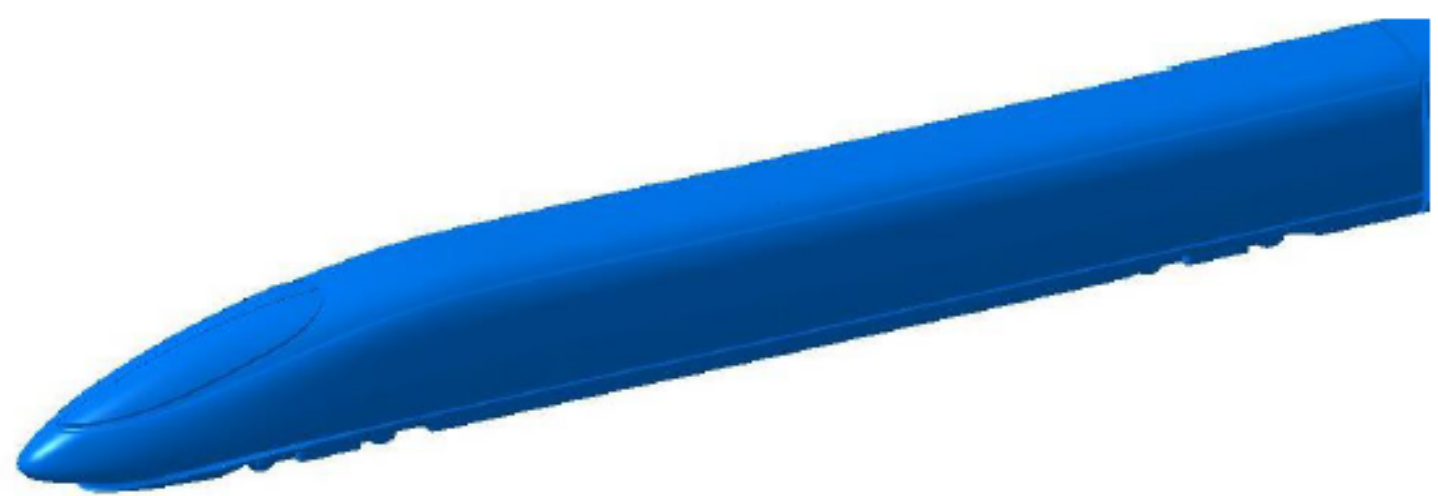

(a) Head car model of CRH-A

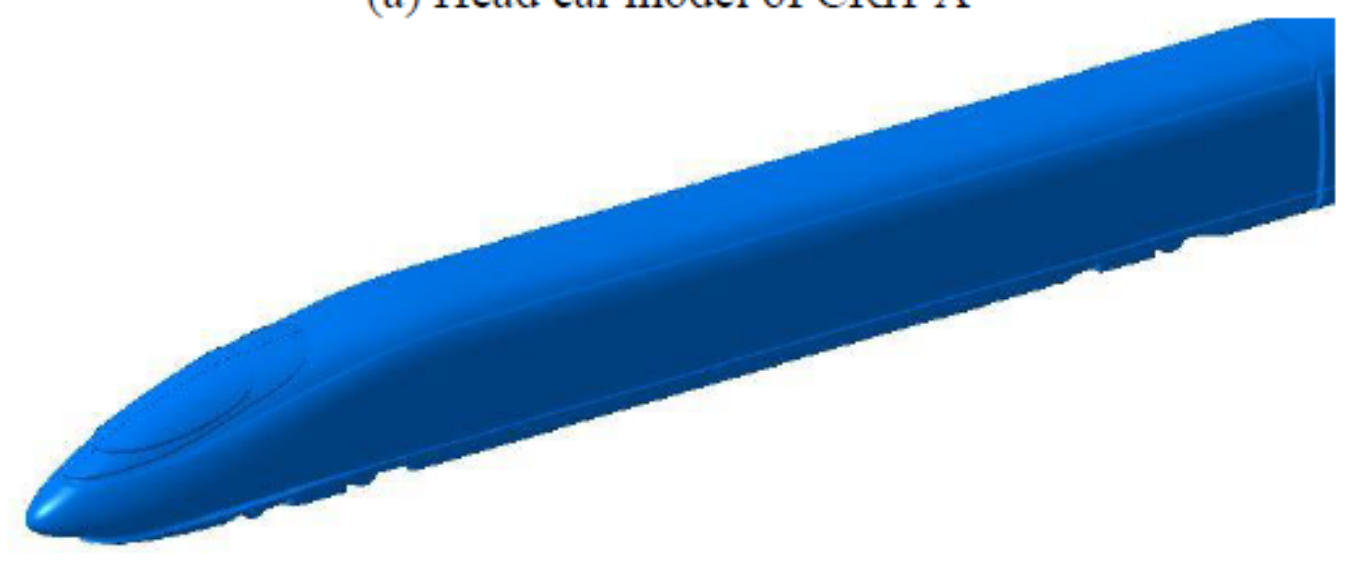

(b) Head car model of CRH-B

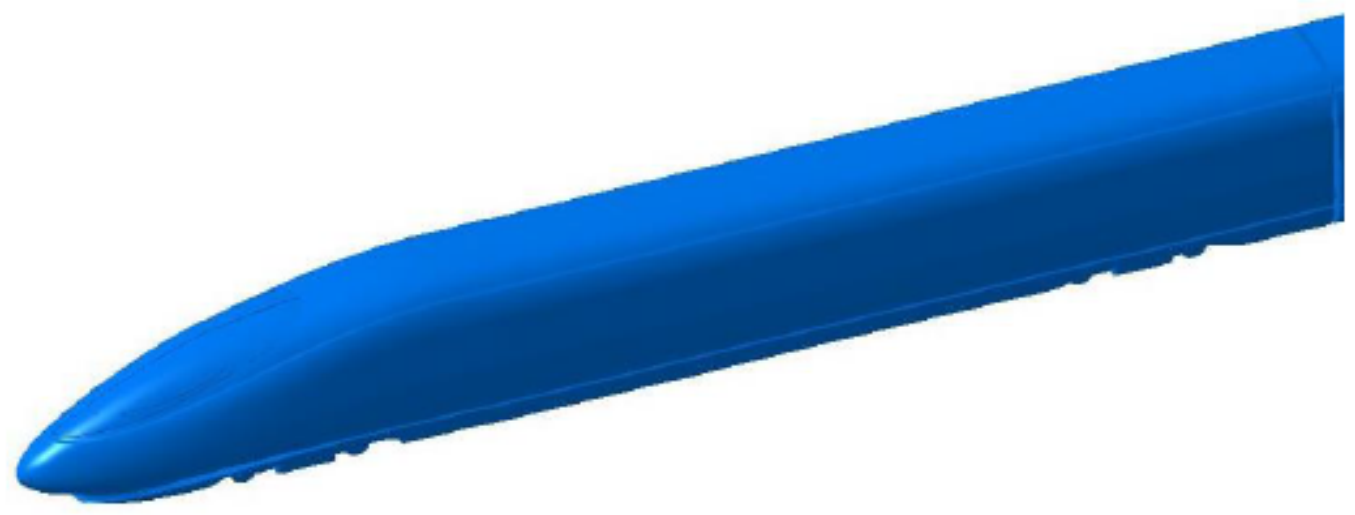

(c) Head car model of $\mathrm{CRH}-\mathrm{C}$

Figure 3

Test models 


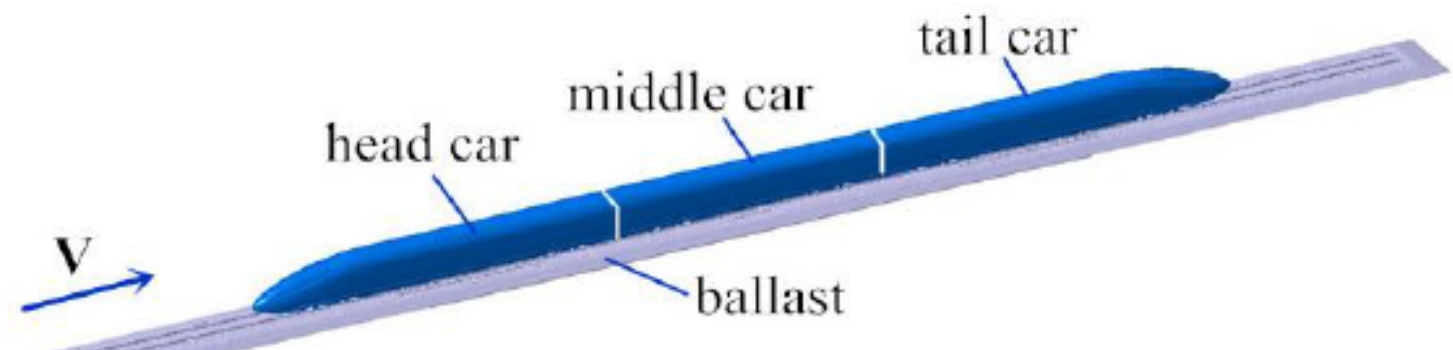

(a) Three-car formation

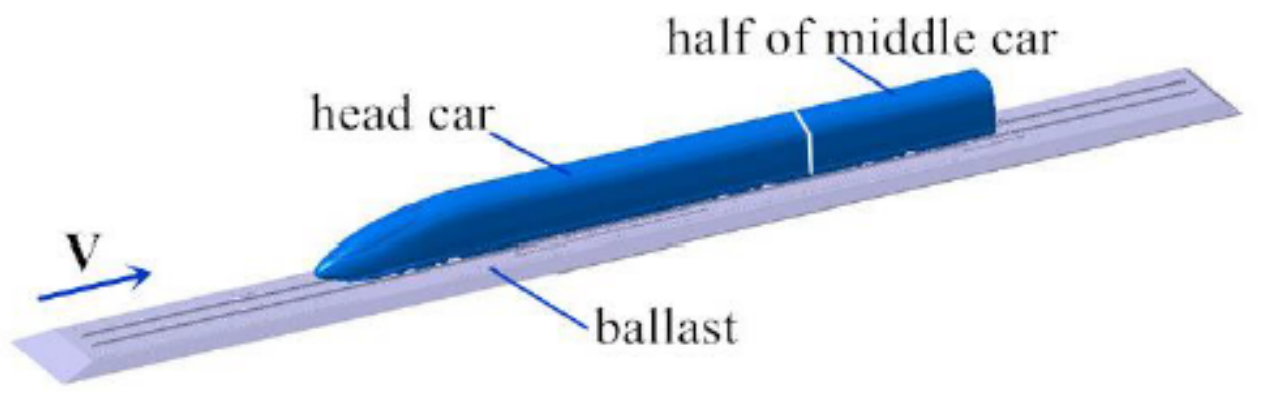

(b) One-and-a-half-car formation

Figure 4

Two different train car formations and the ballast model 


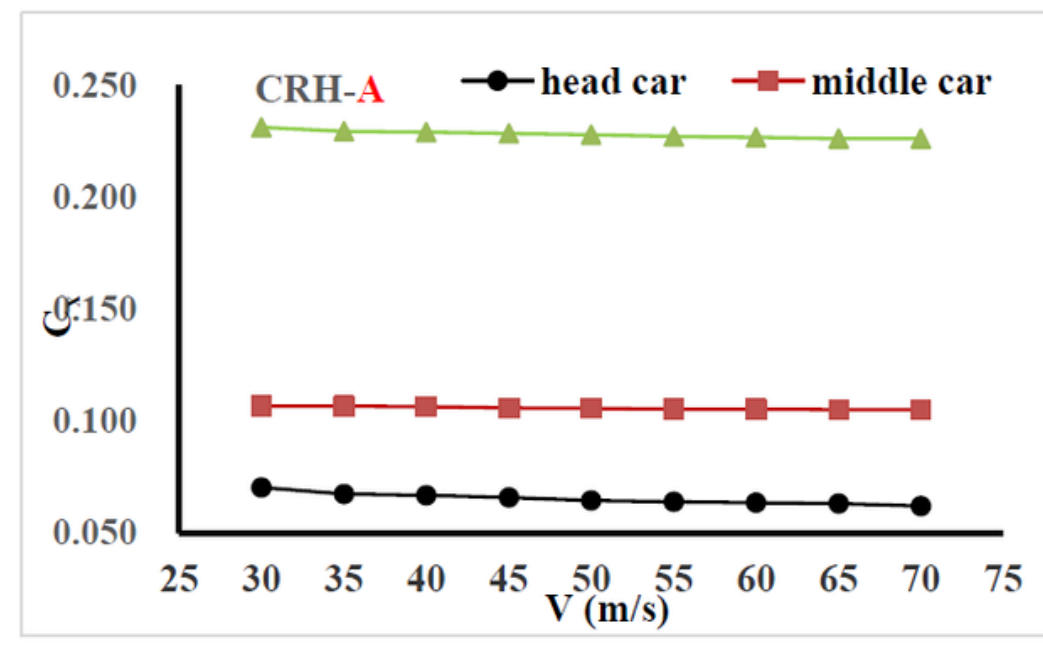

(a)

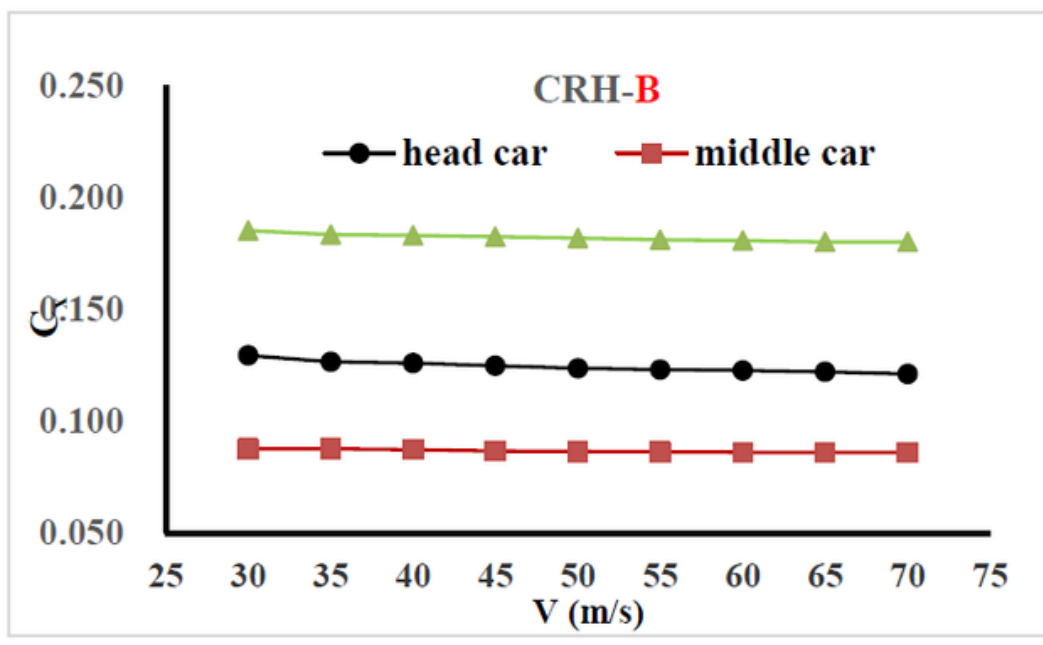

(b)

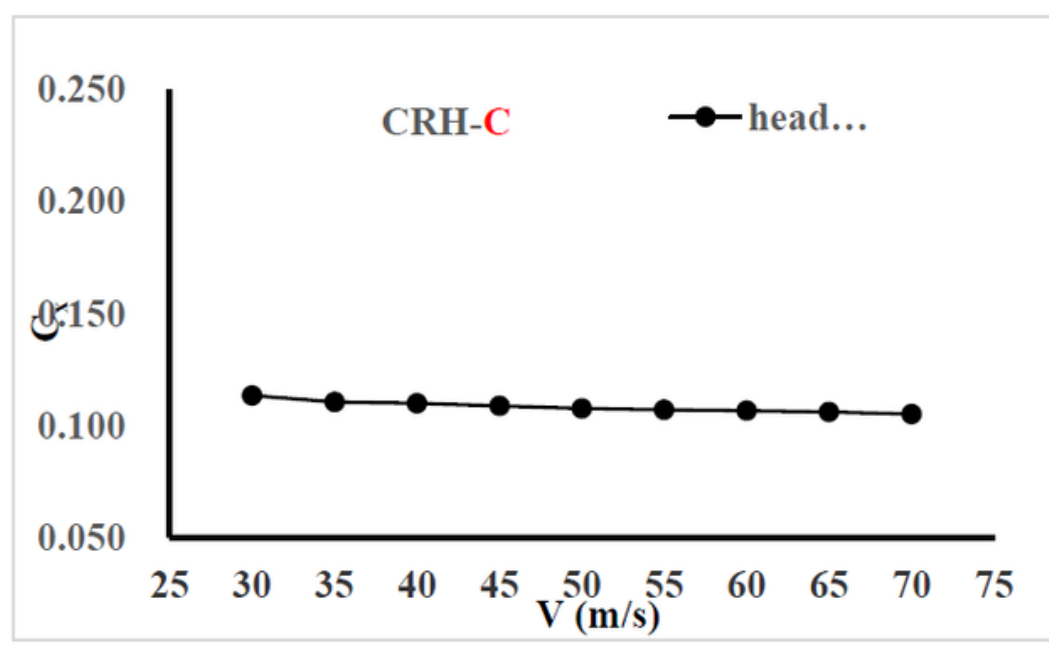

(c)

Figure 5

Changes in drag coefficients of CRH-A, CRH-B, and CRH-C with wind velocity (Reynolds number) 


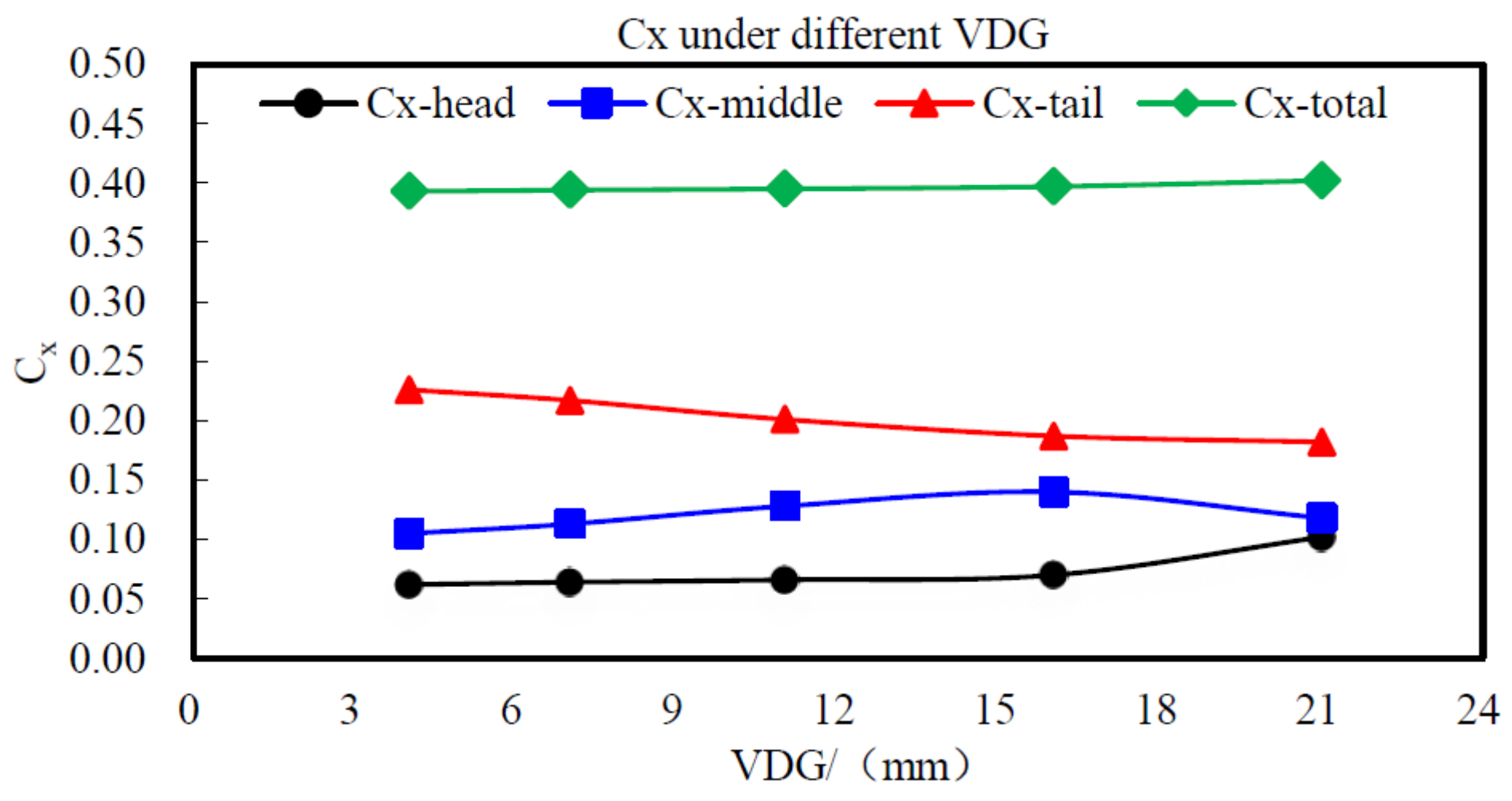

Figure 6

Drag coefficients of each car and total train of $\mathrm{CRH}-\mathrm{A}$ for different vestibule diaphragm gaps 

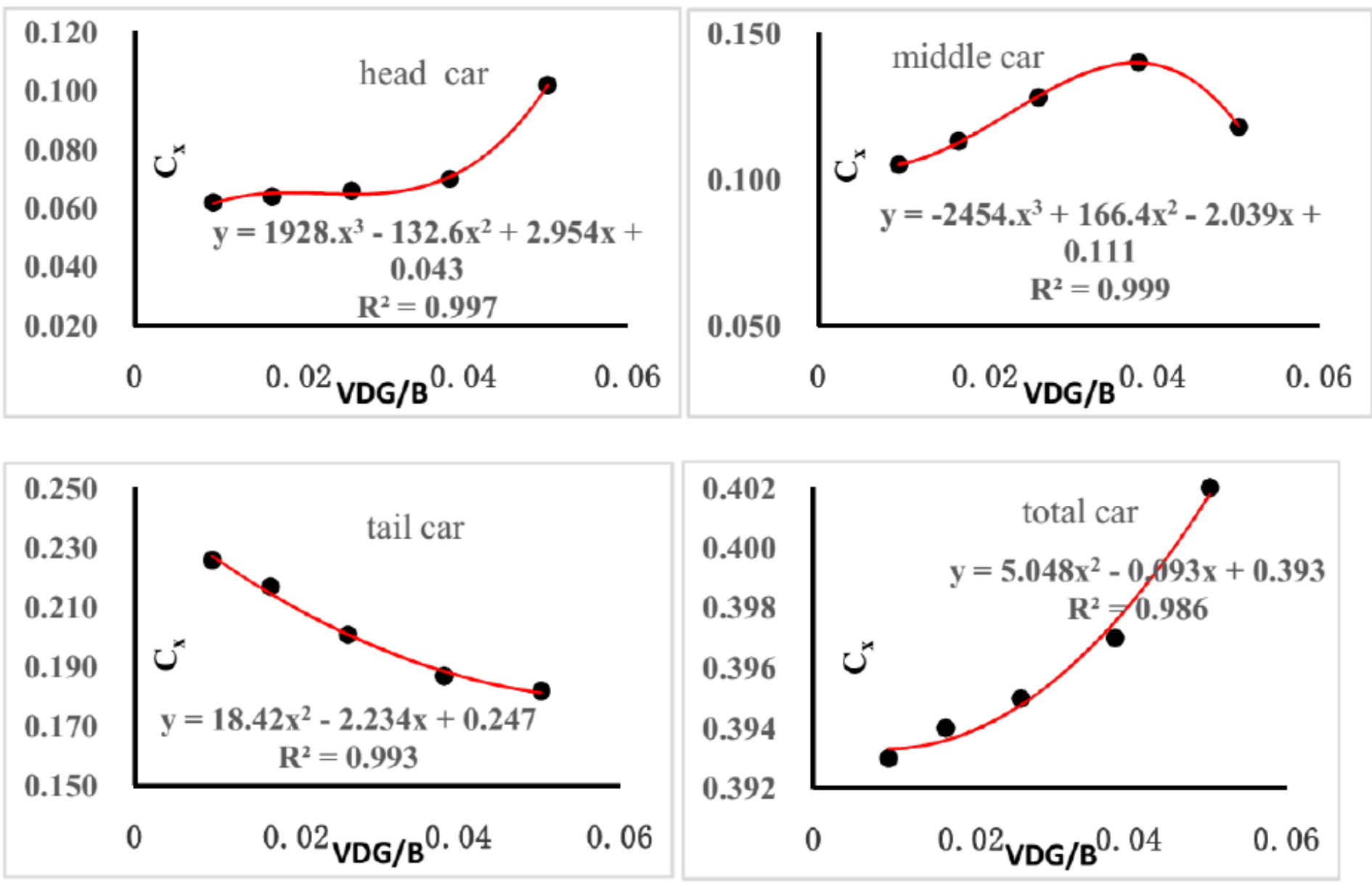

Figure 7

Fitting curves of drag coefficients for different VDGs 


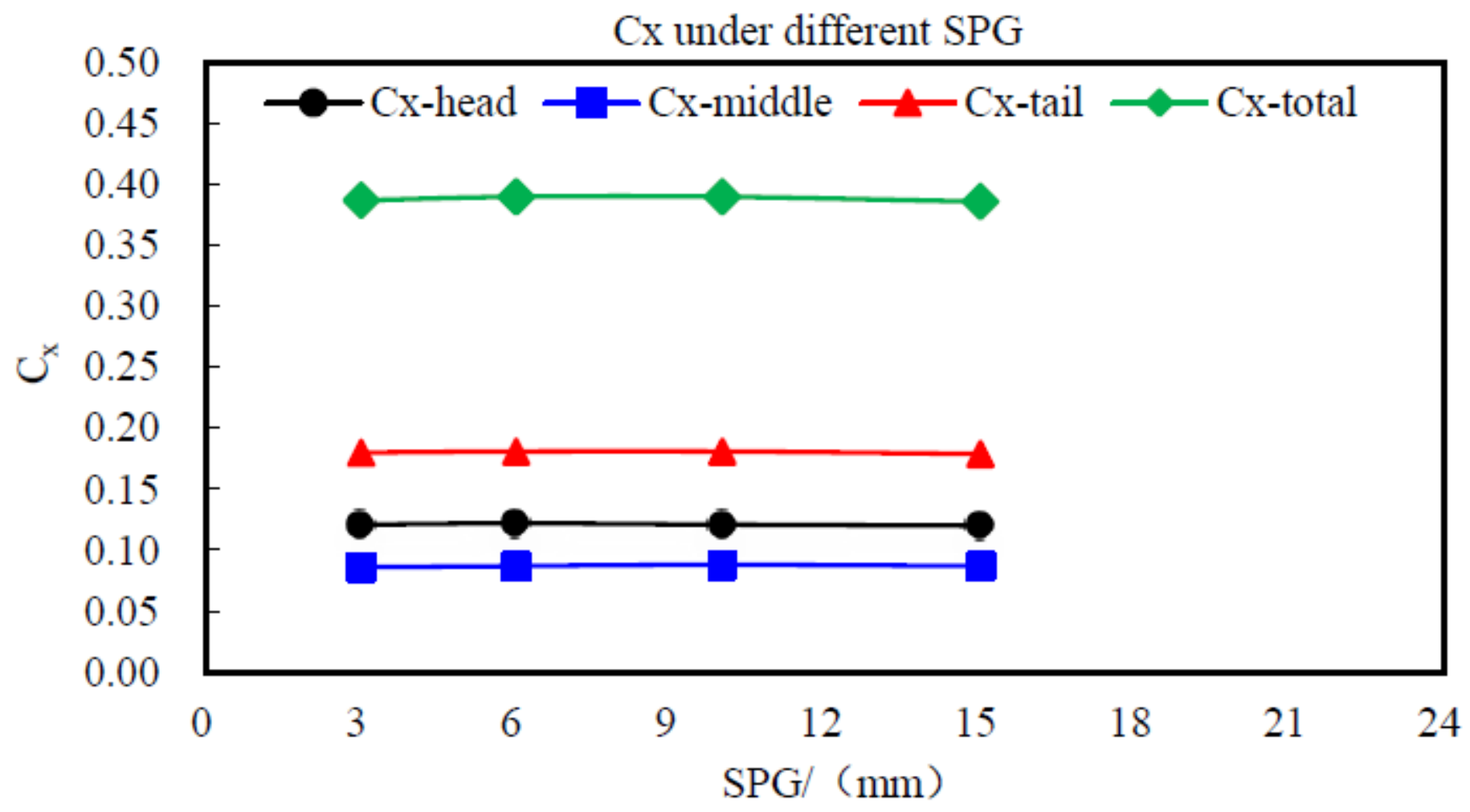

Figure 8

Drag coefficients of each car and total train for different strut-plate gaps

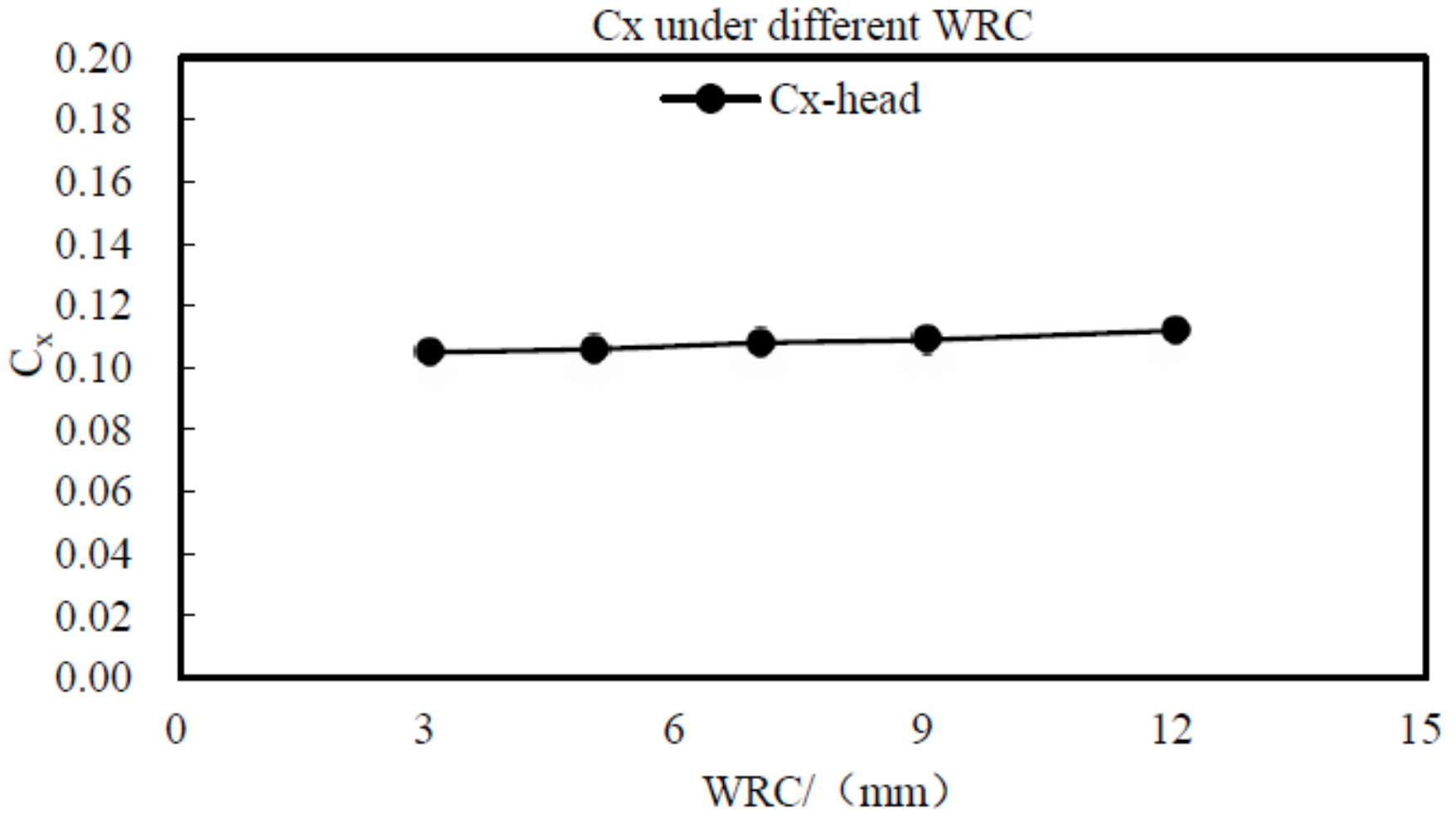

Figure 9

Drag coefficients of head car for different wheel-rail clearance values 